\title{
Exploring central limit theorem on world population density data
}

\begin{abstract}
We do some exploration to Central Limit Theorem on a real dataset. We intend to conduct this study to a real data which has non-normal distribution. Under common sense, it is known that world population density data has right-skewed distribution. A resampling mechanism is done to the original data by varying sample size to study the properties of well-known Central Limit Theorem, such as normality of the sampling distribution and reduction of the standard deviation of sample data due to larger sample size.
\end{abstract}

Keyword: Central limit theorem; Normal distribution; Sample size 